This is a paper submitted to and accepted for publication in:

Beatriz L. Boada, Daniel García-Pozuelo, Maria Jesús L. Boada, and Vicente Díaz. A Constrained Dual Kalman Filter based on pdf truncation for estimation of vehicle parameters and road bank angle: analysis and experimental validation. IEEE Transactions on Intelligent Transportation Systems, vol. 18, no. 4, April 2017. Pp. 1006-1016. https://doi.org/10.1109/TITS.2016.2594217

(C) 2016 IEEE 


\title{
A Constrained Dual Kalman Filter based on pdf truncation for estimation of vehicle parameters and road bank angle: analysis and experimental validation
}

\author{
Beatriz L. Boada, Daniel Garcia-Pozuelo, Maria Jesus L. Boada, and Vicente Diaz
}

\begin{abstract}
Vehicles today are equipped with control systems that improve their handling and stability. Knowledge of road bank angle and vehicle parameters is crucial for good behaviour in this type of control. This paper develops a new method for estimating different states, such as vehicle roll angle and road bank angle, and vehicle parameters. This method combines a Dual Kalman Filter with a probability density function (pdf) truncation method to consider the parameter physical limitations. Experimental results show the effectiveness of the proposed method and demonstrate that the incorporation of parameter constraints improves its estimation accuracy. The proposed method provides an estimation of the parameters and the states' physical meaning and stable values within the real boundary limits in contrast to other estimation methods.
\end{abstract}

Index Terms-Dual Kalman Filter, pdf truncation approach, vehicle dynamics, parameter estimation, state estimation, road bank angle.

\section{INTRODUCTION}

$\mathbf{W}$ ITH the recent advancements in the vehicle industry, driving safety in vehicles is one of the key issues in the design of any vehicle. Nowadays, vehicles are equipped with control systems in order to improve their handling and stability [1] [2] [3] [4] [5]. These control systems need to know the behaviour of vehicle (states), such as the sideslip angle, the yaw rate, the roll angle, among others, [6] [7] [8] during different manoeuvres in order to perform appropriately. Specificall, knowledge of the road bank and vehicle roll angles is useful in lateral and rollover stability control systems.

To improve the vehicle lateral stability, the yaw rate and the sideslip angle are controlled so that they follow their target values. The yaw rate can be directly measured by a yaw rate sensor (gyroscope). In addition, the sideslip angle can be obtained from a dual-antenna GPS, but this is a very expensive technique. For this reason, the value of the sideslip angle is currently estimated by a control system. Some methods estimate the sideslip angle by means of the estimated roll angle [9] [10] [11]. The problem with these methods is that neither of them estimate the vehicle roll angle and the road bank angle separately. In [6], the sideslip angle is estimated considering the vehicle roll angle and road bank separately.

Authors are with Mechanical Engineering Department and Research Institute of Vehicle Safety (ISVA) from Universidad Carlos III de Madrid, Avda. de la Universidad, 30. 28911. Leganes, Madrid. SPAIN. (e-mail: \{bboada,dgramos,mjboada,vdiaz\}@ing.uc3m.es).

Manuscript received April XX, 201X; revised August XX, 20XX.
Nevertheless, the road bank angle estimation algorithm was not experimentally evaluated. It is necessary to highlight the fact that the road bank angle is not only important for the performance of designed vehicle stability control systems, but also prevents their unnecessary activation when the vehicle is on a road bank [12] [13].

The rollover stability control systems are based on lateral load transfer, which is directly related to the vehicle roll angle [1]. To improve the performance of these systems, an accurate measure of the vehicle roll angle is needed. One of the problems is that many commercial sensors provide global information on the roll angle (vehicle roll angle + road bank angle) which cannot be directly used as a control variable to drive the Roll Stability Control (RSC) systems [14]. For this reason, it is necessary to design observers to estimate both variables separately.

Several observers and control systems proposed in the literature are based on mathematical models. In this case, it is necessary to know the vehicle parameters in detail in order to obtain real vehicle response. Some of the vehicle parameters can be easily measured such as the mass, the track width or the wheelbase. However, there are other parameters that are unknown and directly unmeasurable such as roll stiffness, roll damping coefficient tyre cornering stiffness, etc. These parameters are usually estimated through an identificatio process.

Therefore, a good deal of research has been carried out to estimate different vehicle parameters. A TS (Two-Stage) approach has been developed by Garatti and Bittanti [15] to estimate the tyre parameters of the Pacejka model from measurements of the car behaviour. Bolhasani and Azadi [16] use a genetic algorithm optimization method to estimate vehicle parameters such as tyre cornering stiffness, mass moments of inertia about longitudinal and vertical axes, sprung mass and roll stiffness and roll damping coefficient of the suspension system.

Because there is no guarantee that model behaviour does not change over time, the model adjustment through the time variation of parameters together with state variables is crucial. Accordingly, a procedure that can provide the simultaneous estimate of states and parameters is required. There are different methods, which are used to obtain simultaneously the parameter and states estimates for dynamic systems, such as joint state/parameter estimation and dual state/parameter 
estimation. The former method concatenates both states and parameters into a joint state vector. The latter method considers a separate state-space formulation for states and parameters [17]. Using the dual state/parameter estimation, it is possible to switch off the parameter estimator, once a sufficientl good set of estimates for the parameters have been found [18]. This should increase the performance of the state estimator, as it reduces the parameter uncertainties as well as disturbances arising from the varying model parameters. Regarding the joint state/parameter estimates method, it has been known to have convergence problems [17]. Furthermore, if the sensitivity matrix of the state-to-output equations is ill-conditioned, the joint state/parameter estimation method results in deteriorated accuracy for parameters estimation [19].

Some researches use a Dual Kalman Filter (DKF) to identify the vehicle parameters and the vehicle state simultaneously [20]. Hong et al. [21] estimate three inertial parameters: the sprung mass, the yaw moment of inertia and the longitudinal position of the center of gravity using a Dual Unscented Kalman Filter (DUKF). Nada et al. [22] provide a model based estimator using DUKF to enhance the real time performance of the algorithm. The model-based estimator deals with both estimation of vehicle states and vehicle parameters such as tyre road friction coefficien and vehicle mass. Wenzel et al. [18] use a Dual Extended Kalman Filter (DEKF) in order to estimate both vehicle states and vehicle parameters such as vehicle mass, moment of inertia about vertical axis and the distance between the center of gravity and the front axle.

In most physical systems, process parameters and states are normally bounded by their physical limitations. This aspect implies that the estimates of the states and parameters have to satisfy their respective constraints. The incorporation of constraints increases the computational effort of Kalman filter but significantl improves their estimation accuracy.

Different methods have been developed to deal with constraints in Kalman filters The projection method conducts a projection to transform the unconstrained estimate so that the new estimate lies on a constrained surface [23] [24] [25]. In the pdf (probability density function) truncation method, the pdf is taken from the state/parameter estimate, which is calculated by Kalman filter (assuming that it is Gaussian) and truncated at the constraint edges. The constrained state/parameter estimate becomes equal to the mean of the truncated pdf [23]. The pdf truncation approach has been used in different engineering applications: for estimating the pose of a human hand and refinin the $3 \mathrm{D}$ shape of the given hand model from a monocular image sequence which contains no depth data [26], for estimating the aircraft turbofan engine health parameter [27] and for correcting the localization and registration of a surgical robot [28].

The main contribution of the work presented in this paper is a constrained dual state and parameter estimation algorithm using a DKF for analysis of lateral vehicle dynamics. The pdf truncation algorithm is proposed for handling constraints on parameters in order to estimate vehicle parameters and road bank angle. The proposed algorithm retains the advantages of DKF, while being able to systematically handle constraints on parameters. Experimental results show the effectiveness of the proposed algorithm.

This paper is organized as follows. The vehicle model for estimator design is described in Section II. The model considers the road bank angle and vehicle roll angle separately. In Section III, a DKF is designed for estimation of lateral vehicle states and parameters combined with the pdf method. The DKF uses data obtained from sensors mounted on a real vehicle: the sideslip angle, $\beta$, the yaw rate, $r$, the total roll angle, $\phi$ and the roll rate, $\dot{\phi}$. In this case, different problems appear. First of all, the sensor provides global information on the roll angles (road bank angle + vehicle roll angle). Secondly, the number of parameters to estimate is high. Third, the parameters model is a non-linear model. And finall, the number of sensor measurements is low compared with the number of states and parameters to estimate. For these reasons, the solution of this problem is very complex: several solutions can be obtained, even solutions with non-physical meaning. In this case, a constrained method is necessary in order to take into account the known constraints on the parameter vector. In this paper, the pdf method to limit the parameter vector is used. The reason is due to the fact that the possible values of the vehicle parameters are included in well-known ranges and the pdf truncation method is designed for inequality constraints. In Section IV, the real vehicle and sensors mounted on it are described. In addition, the proposed estimator is evaluated using real tests and the results are illustrated. Finally, the summary and conclusions are given in Section V.

\section{Vehicle MODEL WITH ROAD BANK}

In Figure 1, the vehicle roll model with road bank is shown. It is assumed that the vehicle sprung mass rotates around the roll center of the vehicle. The vehicle model has three degrees of freedom (DOF): the sideslip angle $(\beta)$, the yaw rate $(r)$ and the roll angle $\left(\phi_{v}\right)$. The equations of lateral motion for a vehicle model with road bank angle and linear tyre characteristics are [12] [13] [29] [30]:

$$
\begin{aligned}
\dot{\beta}= & -\frac{I_{e q} \cdot C_{0}}{I_{x} \cdot m \cdot V_{x}} \cdot \beta-\left(1+\frac{I_{e q} \cdot C_{1}}{I_{x} \cdot m \cdot V_{x}^{2}} \cdot\right) \cdot r+ \\
& \frac{h_{c r} \cdot\left(m \cdot g \cdot h_{c r}-K_{s u s p}\right)}{I_{x} \cdot V_{x}} \cdot \phi_{v}-\frac{h_{c r} \cdot b}{I_{x} \cdot V_{x}} \cdot \dot{\phi}_{v}+ \\
& \frac{I_{e q} \cdot C_{\alpha f}}{I_{x} \cdot m \cdot V_{x}} \cdot \delta-\frac{g}{V_{x}} \cdot \phi_{r} \\
\dot{r}= & -\frac{C_{1}}{I_{z}} \cdot \beta-\frac{C_{2}}{I_{z} \cdot V_{x}} \cdot r+\frac{a \cdot C_{\alpha f}}{I_{z}} \cdot \delta \\
\ddot{\phi}_{v}= & -\frac{C_{0} \cdot h_{c r}}{I_{x}} \cdot \beta-\left(1+\frac{I_{e q} \cdot C_{1}}{I_{x} \cdot m \cdot V_{x}^{2}}\right) \cdot r+ \\
& \frac{h_{c r} \cdot\left(m \cdot g \cdot h_{c r}-K_{s u s p}\right)}{I_{x} \cdot V_{x}} \cdot \phi_{v}-\frac{C_{s u s p}}{I_{x}} \cdot \dot{\phi}_{v}+ \\
& \frac{C_{\alpha f} \cdot h_{c r}}{I_{x}} \cdot \delta-\dot{p}_{f}
\end{aligned}
$$

where:

$$
\begin{gathered}
C_{0}=C_{\alpha f}+C_{\alpha r} \\
C_{1}=a \cdot C_{\alpha f}-b \cdot C_{\alpha r} \\
C_{2}=a \cdot C_{\alpha f}^{2}+b \cdot C_{\alpha r}^{2} \\
I_{e q}=I_{x}+m \cdot h_{c r}^{2}
\end{gathered}
$$

$m$ is the vehicle mass; $C_{\alpha f}$ and $C_{\alpha r}$ are the cornering stiffness of the front and rear tyre, respectively; $a$ and $b$ are the distances 


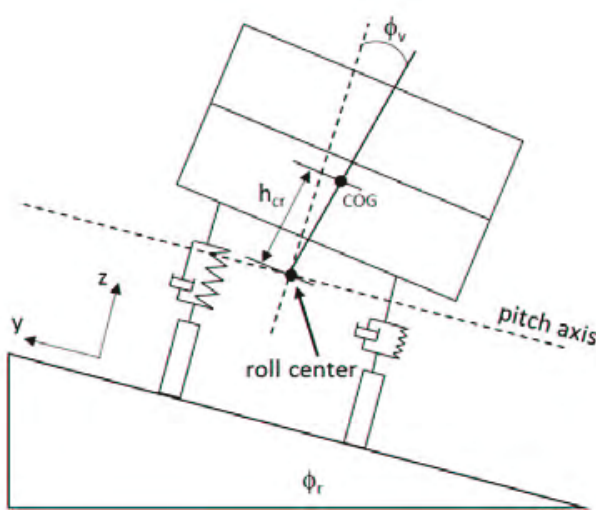

Fig. 1. Vehicle roll model with road bank.

of the front and rear axles from the Center of Gravity (COG); $h_{c r}$ is the distance from roll center to COG; $I_{x}$ is the moment of inertia about the roll axis; $I_{z}$ is the moment of inertia about the yaw axis; $g$ is the acceleration of gravity; $K_{\text {susp }}$ is the roll stiffness, $C_{\text {susp }}$ is the roll damping coefficient $\delta$ is the steering wheel angle; $V_{x}$ is the longitudinal velocity; $p_{f}$ is the $\mathrm{x}$ component of the angular velocity vector of the vehicle frame with respect to the inertial coordinates and $\phi_{r}$ is the road bank angle.

The rate of the road bank angle $\left(\dot{\phi}_{r}\right)$ can be calculated by definin a new variable $\epsilon_{r}$ and assuming the vehicle pitch angle is small [29]:

$$
\dot{\phi}_{r} \approx p_{f}+\varepsilon_{r}
$$

The road bank angle $\left(\phi_{r}\right)$ and the time derivate of $p_{f}$ can be considered as two disturbances to vehicle dynamics. Since the road changes independently, the disturbances due to the road vary independently of the vehicle dynamics. However, the two disturbances are not independent of each other [29]. Therefore, the dynamics of the disturbances can be described as follows assuming that the disturbances due to the road changes are the result of white noise forcing $\ddot{p}_{f}$ and $\dot{\varepsilon}_{r}$ [30]:

$$
\begin{aligned}
\ddot{p}_{f} & =0 \\
\dot{\varepsilon}_{r} & =0
\end{aligned}
$$

\section{PARAMETER AND ROAD BANK ESTIMATION}

The proposed estimator architecture is shown in Figure 2. The estimation process is based on DKF for parameter and state estimation [22] [21] [18] [31]. DKF uses the data obtained from steering wheel sensor, inertial sensor and dual GPS antenna mounted on a real vehicle. However, the number of estimated parameters is so large that it could give false solutions. In this sense, a constrained method based on pdf is used to take into account the known constraints on the parameter vector.

\section{A. Dual Kalman Filter}

The Kalman filte is a mathematical tool used for estimating vehicle motion. This filte includes data with a substantial amount of noise and unobserved states in the system which must be estimated. For this reason a Kalman filterin technique has been employed in this work.

In a general case, a vehicle model can be expressed as:

$$
\dot{x}_{s}=f\left(x_{s}, x_{p}, u\right)+w
$$

where $x_{s}$ represents the variable states, $x_{p}$ represents the variable parameters and $w$ is the process noise vector.

Assuming that the system in Section II is linear, then, the continuous time state space system is:

$$
\begin{gathered}
\dot{x}_{s}=A \cdot x_{s}+B \cdot u+w \\
y=C \cdot x_{s}+v
\end{gathered}
$$

where $x_{s}$ represents the state vector, $\left[\beta, r, \phi_{v}, \dot{\phi}_{v}, \phi_{r}, p_{f}, \epsilon_{r}\right]^{T}$; $\mathrm{u}$ is the input, $\delta ; y$ is the measurement vector, $[\beta, r, \phi, \phi]^{T}$ and $v$ is the measurement noise vector. $w$ and $v$ are assumed to be white, zero mean and uncorrelated:

$$
\begin{aligned}
& w \sim N(0, Q) \\
& v \sim N(0, R)
\end{aligned}
$$

where $Q$ and $R$ are the covariance matrices describing the second-order properties of the state and measurement noise. The values of matrices $A, B$ and $C$ are define in equation 15.

In order to operate with the sensors data, the discrete state space system is obtained:

$$
\begin{aligned}
& x_{s, k}=A_{d} \cdot x_{s, k-1}+B_{d} \cdot u_{k}+w_{k} \\
& y_{k}=C \cdot x_{s, k}+v_{k}
\end{aligned}
$$

where, $x_{s, k}=\left[\beta_{k}, r_{k}, \phi_{v, k}, \dot{\phi}_{v, k}, \phi_{r, k}, p_{f, k}, \epsilon_{r, k}\right]^{T}, A_{d}=(1+$ $\left.T_{s} \cdot A\right), B_{d}=T_{s} \cdot B$ and $T_{s}$ is the sample time. $A_{d}$ and $B_{d}$ are matrices, which depend on the parameter vector, $x_{p}=$ $\left[C_{\alpha f}, C_{\alpha r}, I_{x}, I_{z}, K_{\text {susp }}, C_{\text {susp }}, h_{c r}\right]^{T}$.

In this work, a DKF algorithm has been used to estimate both the parameters of vehicle model and the vehicle states. At every time step $k$, a Kalman state filte estimates the state using the current parameter estimate $\tilde{x}_{p}$, while a Kalman parameter filte estimates the vehicle parameters using the current state estimate $\tilde{x}_{s}$.

The DKF is summarized as the following recursive equations:

1) Parameter prediction:

$$
\begin{gathered}
\tilde{x}_{p, k \mid k-1}=\tilde{x}_{p, k-1 \mid k-1} \\
P_{p, k \mid k-1}=P_{p, k-1 \mid k-1}+Q_{p}
\end{gathered}
$$

2) State prediction:

$$
\begin{gathered}
\tilde{x}_{s, k \mid k-1}=A_{d}\left(\tilde{x}_{p, k \mid k-1}\right) \cdot \tilde{x}_{s, k-1 \mid k-1}+ \\
B_{d}\left(\tilde{x}_{p, k \mid k-1}\right) \cdot u_{k}
\end{gathered}
$$

3) State correction:

$$
\begin{aligned}
& K_{s, k}=P_{s, k \mid k-1}+C^{T} \cdot\left[C \cdot P_{s, k \mid k-1} \cdot C^{T}+R_{s}\right]^{-1} \\
& \tilde{x}_{s, k \mid k}=\tilde{x}_{s, k \mid k-1}+K_{s, k} \cdot\left[y_{\text {obs }}-C \cdot \tilde{x}_{s, k \mid k-1}\right]
\end{aligned}
$$




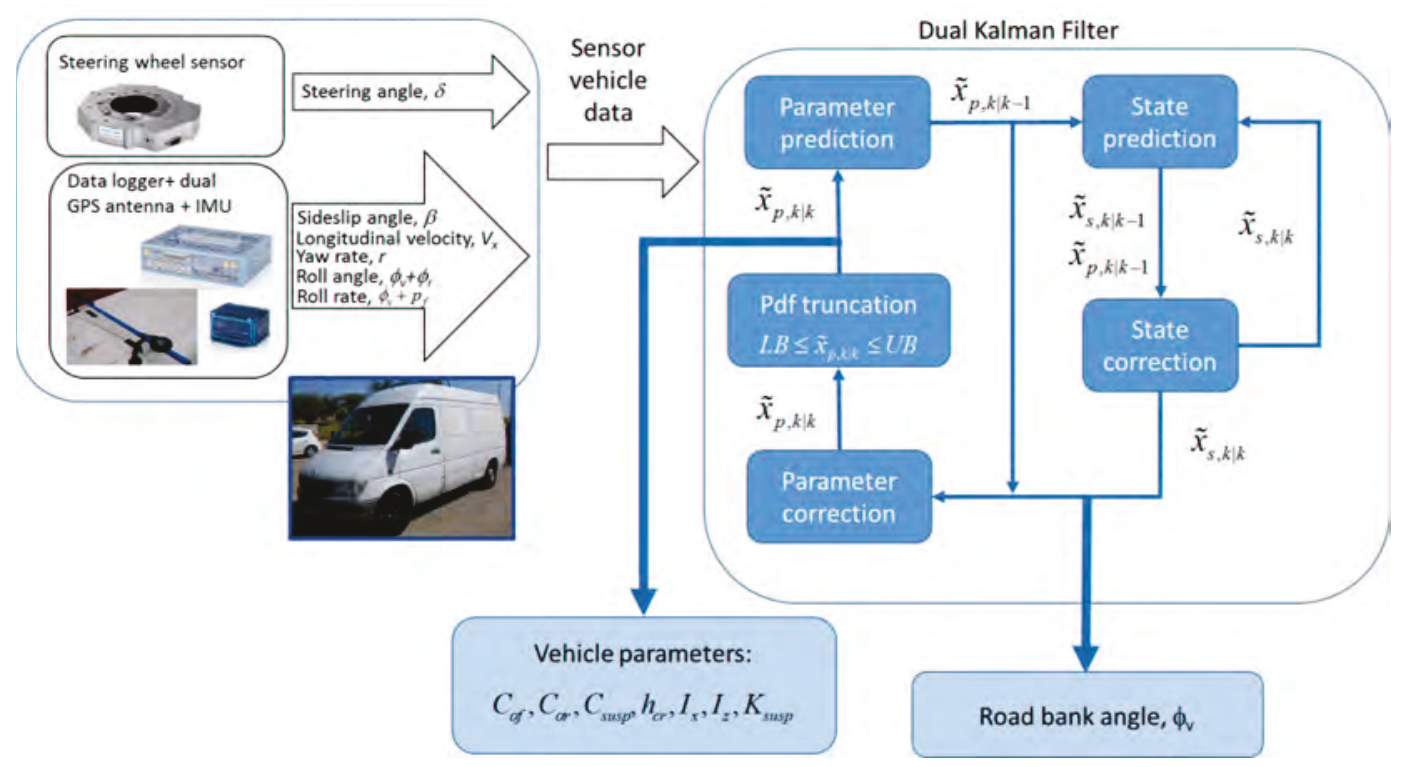

Fig. 2. Estimator architecture.

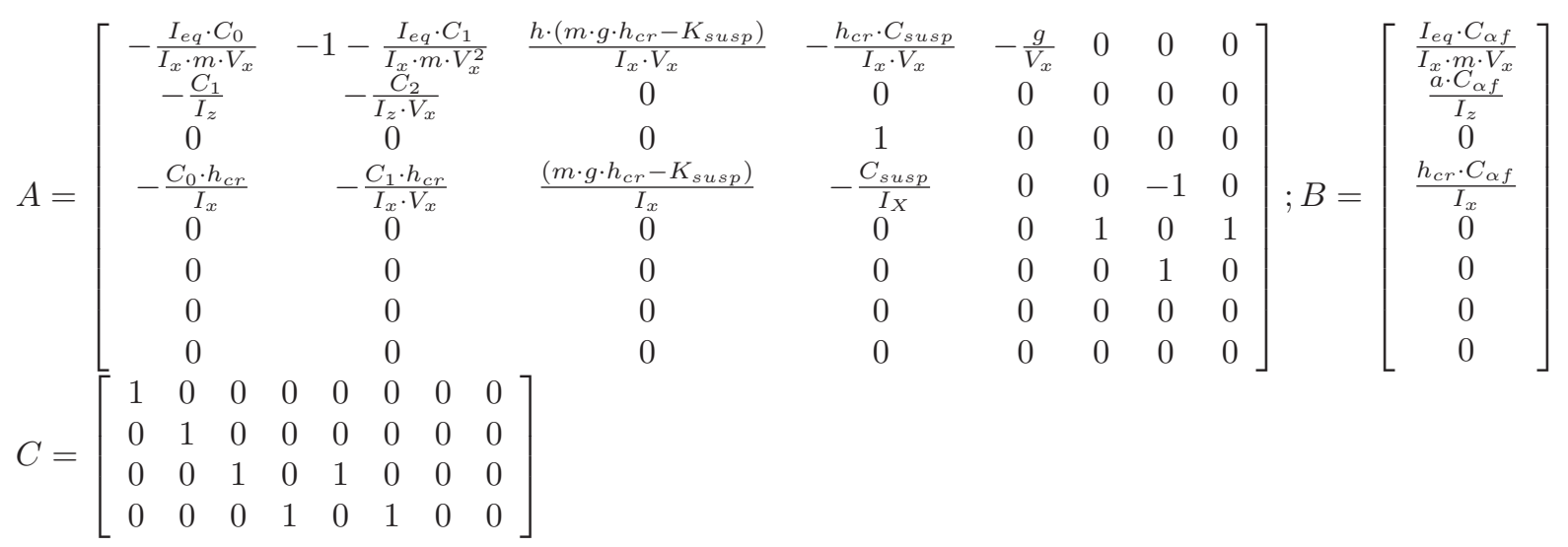

$$
P_{s, k \mid k}=\left[I-K_{s, k} \cdot C\right] \cdot P_{s, k \mid k-1}
$$

4) Parameter correction:

$$
\begin{gathered}
K_{p, k}=P_{p, k \mid k-1}+J^{T} \cdot\left[J \cdot P_{p, k \mid k-1} \cdot J^{T}+R_{p}\right]^{-1} \\
y_{e s t}=\left[\left(A_{d}\left(\tilde{x}_{p, k \mid k-1}\right) \cdot \tilde{x}_{s, k \mid k}+B_{d}\left(\tilde{x}_{p, k \mid k-1}\right) \cdot u\right)\right] \\
\tilde{x}_{p, k \mid k}=\tilde{x}_{p, k \mid k-1}+K_{p, k} \cdot\left[y_{o b s}-y_{e s t}\right] \\
P_{p, k \mid k}=\left[I-K_{p, k} \cdot J\right] \cdot P_{p, k \mid k-1}
\end{gathered}
$$

where $P_{p}$ and $P_{s}$ are the error covariance matrices for parameters and states, respectively. $K_{p}$ and $K_{s}$ are the optimal Kalman gain matrices for parameters and states, respectively.

The DKF depends on the definitio of the four covariance matrices: $R_{p}, R_{s}, Q_{p}$ and $Q_{s}$. Since parameter and state estimators depend on the same output, $y_{o b s}$, the measurements for noise covariances are the same:

$$
R_{s}=R_{p}=\left[\begin{array}{cccc}
\sigma_{\beta}^{2} & 0 & 0 & 0 \\
0 & \sigma_{r}^{2} & 0 & 0 \\
0 & 0 & \sigma_{\phi}^{2} & 0 \\
0 & 0 & 0 & \sigma_{\dot{\phi}}^{2}
\end{array}\right]
$$

The measurements for noise covariances are given by sensor errors [32]: $\sigma_{\beta}=0.01 \mathrm{deg}, \sigma_{r}=0.01 \mathrm{deg} / \mathrm{s}, \sigma_{\phi}=0.1 \mathrm{deg}$ and $\sigma_{\dot{\phi}}=0.01 \mathrm{deg} / \mathrm{s}$.

The process noise covariance matrix of state estimator is given by:

$$
Q_{s}=R_{0} \cdot I
$$

where $R_{0}$ is a large value [18]. In our case, $R_{0}$ takes the value 100,000 . The process noise covariance matrix of parameter estimator is given by:

$$
Q_{p}=\operatorname{diag}\left[\sigma_{C_{\alpha f}}^{2}, \sigma_{C_{\alpha r}}^{2}, \sigma_{I_{x}}^{2}, \sigma_{I_{z}}^{2}, \sigma_{K_{s u s p}}^{2}, \sigma_{C_{s u s p}}^{2}, \sigma_{h_{c r}}^{2}\right]
$$

The variables $\sigma_{C_{\alpha f}}, \sigma_{C_{\alpha r}}, \sigma_{I_{x}}, \sigma_{I_{z}}, \sigma_{K_{s u s p}}, \sigma_{C_{s u s p}}, \sigma_{h_{c r}}$ are of an order of $1 \%$ of the corresponding initial values of parameters [18]: $C_{\alpha f}, C_{\alpha r}, I_{x}, I_{z}, K_{\text {susp }}, C_{\text {susp }}, h_{c r} . \mathrm{J}$ is the Jacobian matrix of parameter estimator. This matrix can be written as is given in equation 31 .

\section{B. Probability Density Function truncation approach}

In most physical systems, their parameters and states are normally bounded between their physical limits. This implies 
$J=\left.\frac{\partial f\left(x_{s}, x_{p}\right)}{\partial x_{p}}\right|_{\bar{x}_{s, k}, \bar{x}_{p, k}}=\left[\begin{array}{ccccccc}\frac{\partial \beta_{k}}{\partial C_{\alpha f}} & \frac{\partial \beta_{k}}{\partial C_{\alpha r}} & \frac{\partial \beta_{k}}{\partial I_{x}} & \frac{\partial \beta_{k}}{\partial I_{z}} & \frac{\partial \beta_{k}}{\partial K_{s u s p}} & \frac{\partial \beta_{k}}{\partial C_{s u s p}} & \frac{\partial \beta_{k}}{\partial h_{c r}} \\ \frac{\partial r_{k}}{\partial C_{\alpha f}} & \frac{\partial r_{k}}{\partial C_{\alpha r}} & \frac{\partial r_{k}}{\partial I_{x}} & \frac{\partial r_{k}}{\partial I_{z}} & \frac{\partial r_{k}}{\partial K_{s u s p}} & \frac{\partial r_{k}}{\partial C_{s u s p}} & \frac{\partial r_{k}}{\partial h_{c r}} \\ \frac{\partial\left(\phi_{v, k}+\phi_{r, k}\right)}{\partial C_{\alpha f}} & \frac{\partial\left(\phi_{v, k}+\phi_{r, k}\right)}{\partial C_{\alpha r}} & \frac{\partial\left(\phi_{v, k}+\phi_{r, k}\right)}{\partial I_{x}} & \frac{\partial\left(\phi_{v, k}+\phi_{r, k}\right)}{\partial I_{z}} & \frac{\partial\left(\phi_{v, k}+\phi_{r, k}\right)}{\partial K_{s u s p}} & \frac{\partial\left(\phi_{v, k}+\phi_{r, k}\right)}{\partial C_{s u s p}} & \frac{\partial\left(\phi_{v, k}+\phi_{r, k}\right)}{\partial h_{c r}} \\ \frac{\partial\left(\dot{\phi}_{v, k}+p_{f, k}\right)}{\partial C_{\alpha f}} & \frac{\partial\left(\dot{\phi}_{v, k}+p_{f, k}\right)}{\partial C_{\alpha r}} & \frac{\partial\left(\dot{\phi}_{v, k}+p_{f, k}\right)}{\partial I_{x}} & \frac{\partial\left(\dot{\phi}_{v, k}+p_{f, k}\right)}{\partial I_{z}} & \frac{\partial\left(\dot{\phi}_{v, k}+p_{f, k}\right)}{\partial K_{s u s p}} & \frac{\partial\left(\dot{\phi}_{v, k}+p_{f, k}\right)}{\partial C_{s u s p}} & \frac{\partial\left(\dot{\phi}_{v, k}+p_{f, k}\right)}{\partial h_{c r}}\end{array}\right]$

that the estimation of the parameters and states has to satisfy their respective constraints. To consider this fact, the DKF has to be modifie in order to incorporate the constraints. In this work, only parameter constraints are considered. States can be also constrained but this implies more computational effort and achieved results (see Section IV) show that it is not necessary to take them into account. The parameters should stay within certain positive ranges:

$$
L B \leq\left[C_{\alpha f}, C_{\alpha r}, I_{x}, I_{z}, K_{\text {susp }}, C_{\text {susp }}, h_{c r}\right] \leq U B
$$

where vectors $L B$ and $U B$ represent the lower and upper bounds of the vehicle parameters, respectively. The proposed constrained dual estimation is based on pdf truncation algorithm [23] [33] [27]. In this approach, the pdf, which is calculated by the Kalman filte, is taken and truncated at the constraint edge. The constrained parameters become equal to the mean of the truncated pdf. The DKF estimate $\tilde{x}_{p}(k)$ is a Gaussian random variable with a mean of $x_{p}(k)$ and a covariance of $P_{p, k \mid k}$.

Suppose that at time $k, p$ scalar parameter constraints are given:

$$
a_{k, i} \leq D_{k, i}^{T} \cdot \tilde{x}_{p, k \mid k} \leq b_{k, i} \quad \mathrm{i}=1, \ldots, \mathrm{p}
$$

where $a_{k, i}<b_{k, i}$. This is a two-sided constraint on the linear function of the parameter $D_{k, i}^{T} \cdot \tilde{x}_{p, k \mid k}$. Now suppose at time $k$ that we have a Kalman Filter estimate $x_{p}$ with covariance $P_{p, k \mid k}$. The problem is to truncate the Gaussian pdf $N\left(\tilde{x}_{p, k \mid k}, P_{p, k \mid k}\right)$ at the $p$ constraints, and then fin the mean $\tilde{x}_{p, k \mid k}$ and covariance $P_{p, k \mid k}$ of the truncated pdf.

Because of the difficult of computing the distribution truncated with all parameter constraints, the algorithm is approximated by a sequential truncation with each single constraint.

To compute $\left(\tilde{x}_{p, k \mid k}, P_{p, k \mid k}\right)$ given the associated $i-t h$ parameter constraint in equation 33, the firs step is to initialize:

$$
\begin{aligned}
& i=0 \\
& \tilde{x}_{p, i}(k)=\tilde{x}_{p, k \mid k} \\
& P_{p, i}(k)=P_{p, k \mid k}
\end{aligned}
$$

We perform the transformation:

$$
z_{k, i}=S_{i} \cdot W_{i}^{-1 / 2} \cdot T_{i}^{T} \cdot\left(x_{p, i}(k)-\tilde{x}_{p, i}(k)\right)
$$

$T_{i}$ and $W_{i}$ are obtained from the Jordan canonical decomposition of $P_{p, i}(k)$, which satisfies

$$
T_{i} \cdot W_{i} \cdot T_{i}^{T}=P_{p, i}(k)
$$

$S_{i}$ is an orthogonal matrix obtained by using Gram-Schmidt orthogonalisation that satisfie [23]:

$S_{i} \cdot W_{i}^{1 / 2} \cdot T_{i}^{T} \cdot D_{i}(k)=\left[\left(D_{i}^{T}(k) \cdot P_{p . i}(k) \cdot D_{i}(k)\right)^{1 / 2} \quad 0 \quad \ldots \quad 0 \quad\right]^{T}$
From equations 35,36 and 37 , it is shown that vector $z_{k, i}$ has a mean 0 and covariance matrix of identity, and the constraint is transformed in a normalized scalar constraint:

$$
c_{k, i} \leq\left[\begin{array}{llll}
1 & 0 & \cdots & 0
\end{array}\right] \cdot z_{k, i} \leq d_{k, i}
$$

where:

$$
c_{k, i}=\frac{a_{k, i}-\left(D_{i}^{T}(k) \cdot \tilde{x}_{p, i}(k)\right)}{\left(D_{i}^{T}(k) \cdot P_{p, i}(k) \cdot D_{i}(k)\right)^{1 / 2}}
$$

and

$$
d_{k, i}=\frac{b_{k, i}-\left(D_{i}^{T}(k) \cdot \tilde{x}_{p, i}(k)\right)}{\left(D_{i}^{T}(k) \cdot P_{p . i}(k) \cdot D_{i}(k)\right)^{1 / 2}}
$$

Since $z_{k, i}$ has an identity covariance matrix, its elements are statistically independent of one another. Only the firs element of $z_{k, i}$ is constrained, so the pdf truncation reduces to a onedimensional truncation.

We normalize the truncated pdf so that it has an area of one, and the firs element of $z_{k, i}$ is constrained between $c_{k, i}$ and $d_{k, i}$ :

$$
\begin{aligned}
& \operatorname{pdf}(\zeta)=\left\{\begin{array}{cc}
\alpha_{i} \cdot \exp \left(-\zeta^{2} / 2\right) & \zeta \in\left[c_{k, i}, d_{k, i}\right] \\
0 & \text { otherwise } \\
\sqrt{2} &
\end{array}\right. \\
& \alpha_{i}=\frac{\sqrt{\pi} \cdot\left[\operatorname{erf}\left(d_{k, i} / \sqrt{2}\right)-\operatorname{erf}\left(c_{k, i} / \sqrt{2}\right)\right]}{}
\end{aligned}
$$

where $\operatorname{erf}(\cdot)$ represents the error function.

In this case, the truncated mean $\mu_{i}$ and covariance $\sigma_{i}^{2}$ are computed as:

$$
\begin{aligned}
\mu_{i}= & \alpha_{i} \cdot \int_{c_{k, i}}^{d_{k, i}} \zeta \cdot \exp \left(-\zeta^{2} / 2\right) \cdot d \zeta \\
= & \alpha_{i} \cdot\left[\exp \left(-c_{k, i}^{2} / 2\right)-\exp \left(-d_{k, i}^{2} / 2\right)\right] \\
\sigma_{i}^{2}= & \alpha_{i} \cdot \int_{c_{k, i}}^{d_{k, i}}\left(\zeta-\mu_{i}\right)^{2} \cdot \exp \left(-\zeta^{2} / 2\right) \cdot d \zeta \\
= & \alpha_{i} \cdot\left[\exp \left(-c_{k, i}^{2} / 2\right) \cdot\left(c_{k, i}-2 \mu_{i}\right)-\right. \\
& \left.\exp \left(-d_{k, i}^{2} / 2\right) \cdot\left(d_{k, i}-2 \mu_{i}\right)\right]+\mu_{i}^{2}+1
\end{aligned}
$$

We can compute the mean and variance of the firs element of $z_{k, i}$ after constraint enforcement as:

$$
\begin{gathered}
\tilde{z}_{k, i}=\left[\begin{array}{llll}
\mu_{i} & 0 & \ldots & 0
\end{array}\right]^{T} \\
\operatorname{cov}\left(\tilde{z}_{k, i}\right)=\operatorname{diag}\left(\sigma_{i}^{2}, 1, \ldots, 1\right)
\end{gathered}
$$

where $\operatorname{diag}(a, b, \ldots)$ represents a diagonal matrix whose diagonal elements are $a, b, \ldots$

The mean and variance of the parameter state, after enforcement of the firs constraint, are obtained taking the inverse of the transformation of equation 35 :

$$
\tilde{x}_{p, i+1}(k)=T \cdot W^{1 / 2} \cdot S_{i}^{T} \cdot \tilde{z}_{k, i}+\tilde{x}_{p, i}(k)
$$

$P_{p, i+1}(k)=T \cdot W^{1 / 2} \cdot S_{i}^{T} \cdot \operatorname{cov}\left(\tilde{z}_{k, i}\right) \cdot S_{i} \cdot W^{1 / 2} \cdot T^{T}$ 


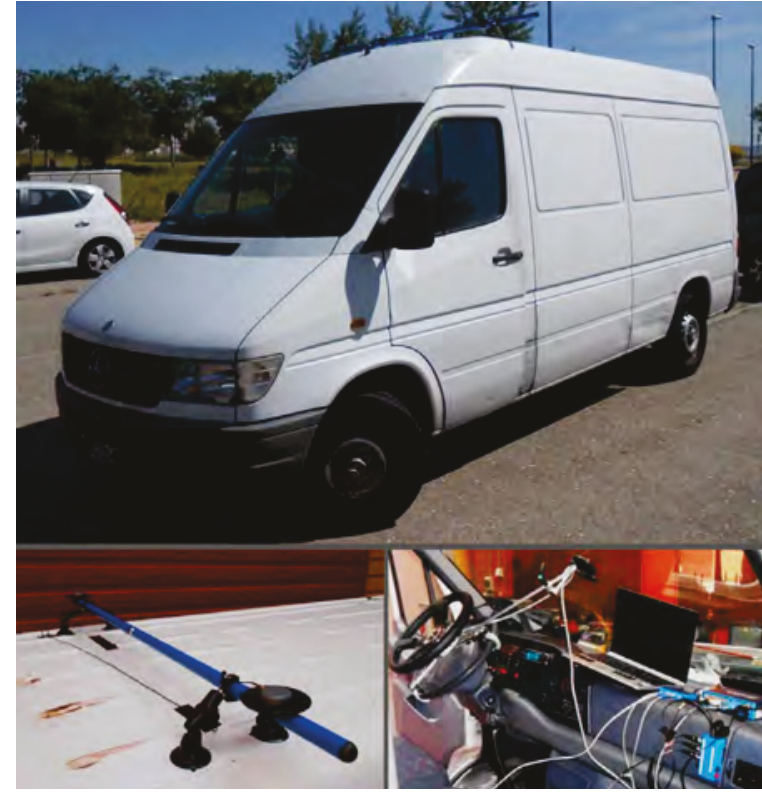

Fig. 3. Experimental setup on a test vehicle (Mercedes Sprinter).

Then, $i$ is incremented by one and the process is repeated from equations $35-47$ to obtain the parameter estimate after enforcement of the next constraint. After going through this process $p$ times, once for each constraint, the fina constrained parameter estimate and covariance at time $\mathrm{k}$ is given by:

$$
\begin{aligned}
& \tilde{x}_{p, k \mid k}=\tilde{x}_{p, p}(k) \\
& P_{p, k \mid k}=P_{p, p}(k)
\end{aligned}
$$

\section{EXPERIMENTAL RESUlTS AND DisCUSSION}

The vehicle used for this research was a Mercedes Sprinter as shown in Figure 3. It was equipped with a steering angle sensor MSW $250 \mathrm{Nm}$ from Kistler, a Vbox 3i dual-antenna data logger, an IMU (Inertial Measurement Unit) sensor and two GPS antennas from Raceologic.

The IMU was mounted on the vehicle floo near its COG. The two antennas were mounted 90 deg to the true heading of the vehicle on the roof in order to measure the sideslip and roll angles with high precision [32].

The data obtained from sensors mounted in the vehicle are: steering wheel, $\delta$, yaw rate, $r$, sideslip angle, $\beta$, roll angle, $\phi_{r}+\phi_{v}$, and roll rate, $\dot{\phi}_{v}+p_{f}$ as depicted in Figure 2 .

The performance of the proposed estimator has been proved for a real vehicle travelling on a dry pavement under different manoeuvres. Straight line, J-turn and slalom manoeuvres are shown in Figures 4 and 5. The speed profil is depicted in Figure 6. From point $\mathrm{A}$ to point $\mathrm{B}$, a straight line test is conducted during 24 seconds; from point $\mathrm{B}$ to point $\mathrm{C}$ a J-turn test is conducted during 30 seconds; from point $\mathrm{C}$ to point $\mathrm{D}$, a slalom test is conducted during 31 seconds; from point $\mathrm{D}$ to point $\mathrm{E}$, a J-turn test is conducted during 27 seconds; and finall, from point $\mathrm{E}$ to point $\mathrm{F}$, a slalom test is conducted during 35 seconds.

In order to demonstrate the improvement provided by the proposed algorithm, its results have been compared with

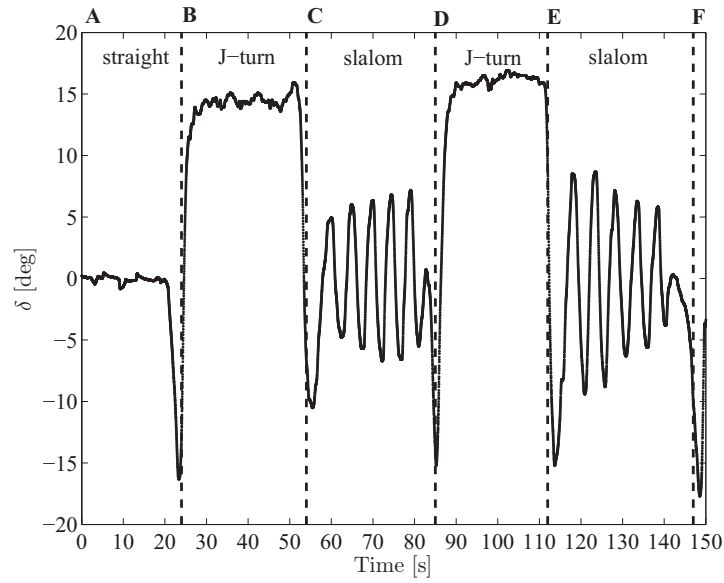

Fig. 4. Vehicle steering wheel angle measures with the sensor MSW $250 \mathrm{Nm}$ during experimental test.

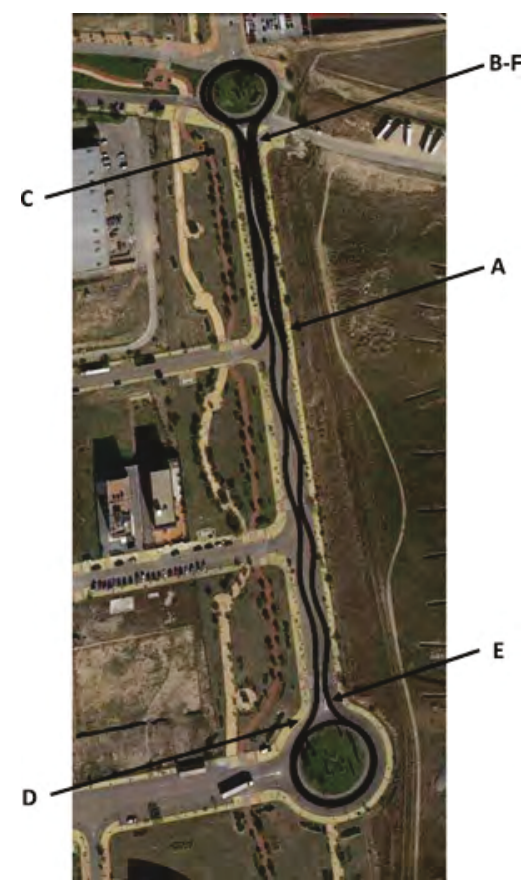

Fig. 5. Experimental test: slalom and J-turn manoeuvres.

other estimators: an estimator without considering parameters constraints and an estimator which uses a modifie projection method in order to take into account the parameters inequalities constraints [27]. Following, the modifie projection method is described. Suppose that we have $p$ inequalities constraints, and $q$ of the $p$ inequalities are active (they are out of boundaries). Denote by $\hat{D}_{k, i}$ the $q$ rows of $D_{k, i}$ that correspond to the active constraints, and denote by $d$ the q components of $L B$ and $U B$ that correspond to the active constraints. Then, the inequality-constrained problem is equivalent to an equality-constrained problem in form of:

$$
\hat{D}_{k, i}^{T} \cdot \tilde{x}_{p, k \mid k}=d \quad \mathrm{i}=1, \ldots, \mathrm{q}
$$




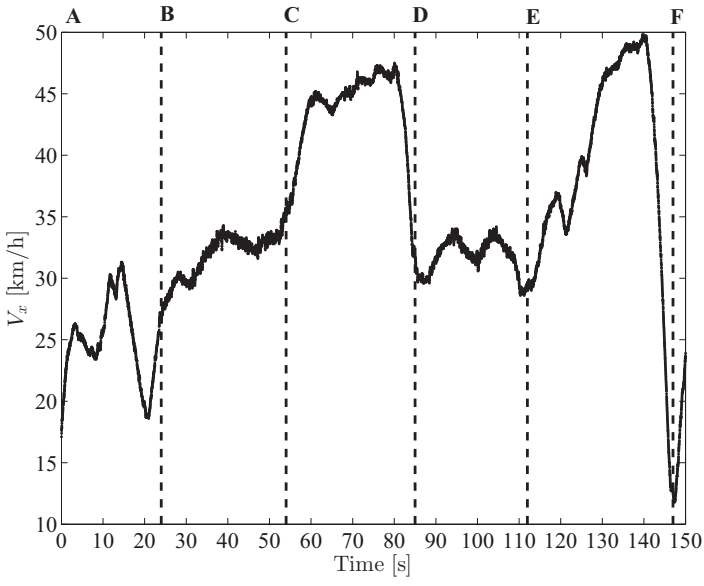

Fig. 6. Vehicle longitudinal speed, $V_{x}$, during experimental test.

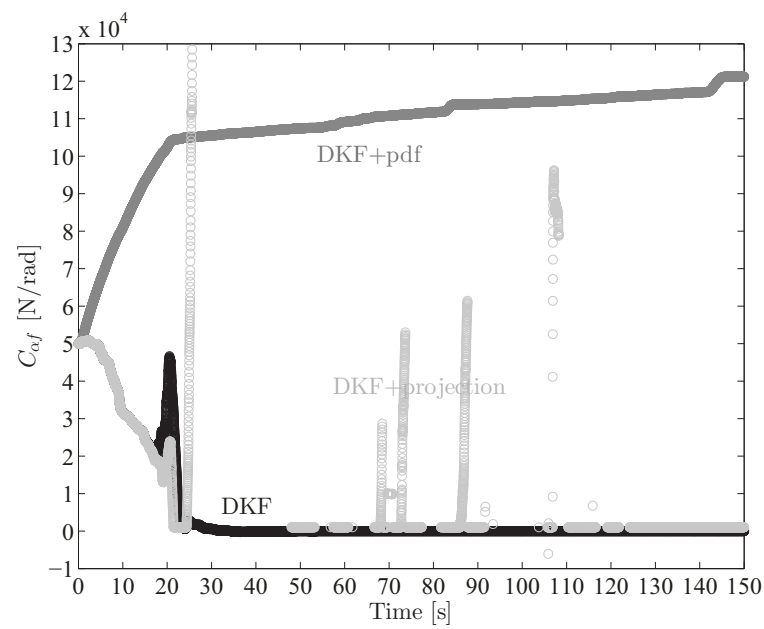

Fig. 7. Front cornering stiffness tyre estimation: DKF without constraints (black), DKF + modifie projection method (light grey) and DKF + pdf truncation method (dark grey).

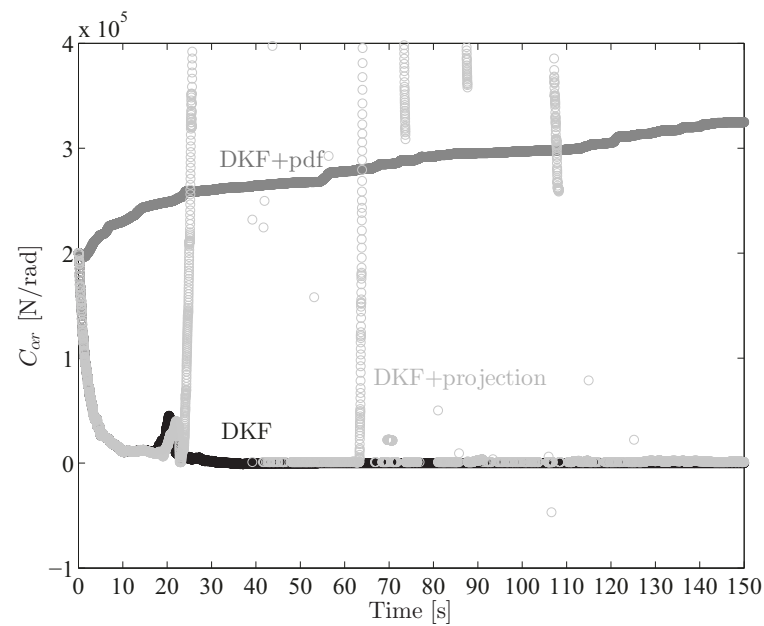

Fig. 8. Rear cornering stiffness tyre estimation: DKF without constraints (black), DKF + modifie projection method (light grey) and DKF + pdf truncation method (dark grey).

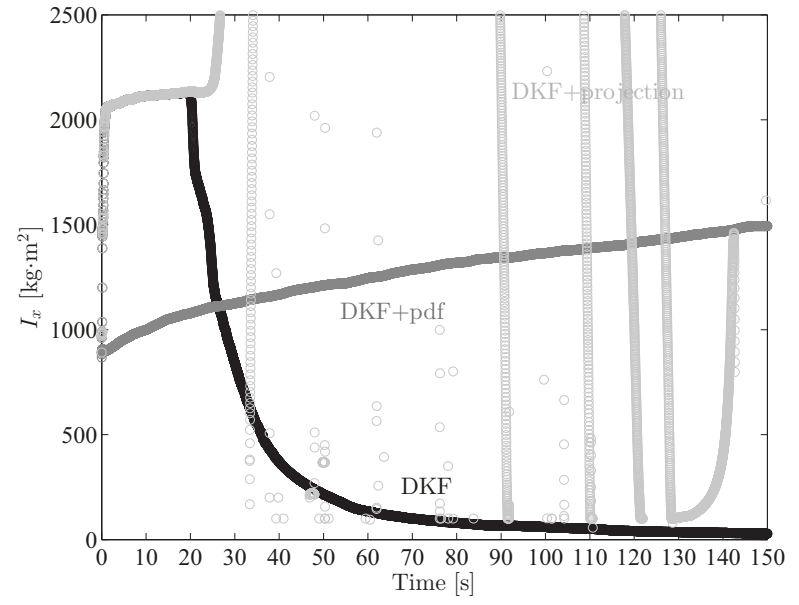

Fig. 9. Moment of inertia about the roll axis estimation: DKF without constraints (black), DKF + modifie projection method (light grey) and DKF + pdf truncation method (dark grey)

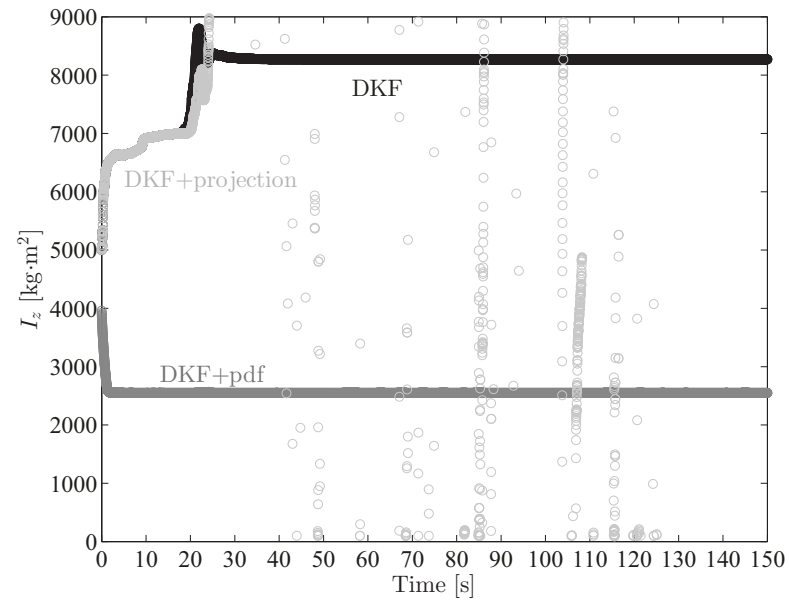

Fig. 10. Moment of inertia about the yaw axis estimation: DKF without constraints (black), DKF + modifie projection method (light grey) and DKF + pdf truncation method (dark grey).

The solution to the above problem is:

$$
\begin{gathered}
\hat{x}_{p, i+1}(k)=\tilde{x}_{p, i}-P_{p, i} \cdot \hat{D}_{k, i}^{T} \cdot \operatorname{inv}\left(\hat{D}_{k, i} \cdot P_{p, i} \cdot \hat{D}_{k, i}^{T}\right) . \\
\left(\hat{D}_{k, i} \cdot \tilde{x}_{p, i}-d\right)
\end{gathered}
$$

For all approaches, identical values of the initial state and parameters were used. The initial values of states are $\left[\beta_{k}, r_{k}, \phi_{v, k}, \dot{\phi}_{v, k}, \phi_{r, k}, p_{f, k}, \epsilon_{r, k}\right]=$ $[0,0,0,0,0,0,0]$. The initial values of parameters are $\left[C_{\alpha f}, C_{\alpha r}, I_{x}, I_{z}, K_{\text {susp }}, C_{\text {susp }}, h_{c r}\right]=\left[5 \cdot 10^{4}\right.$ $\mathrm{N} / \mathrm{rad}, 2 \cdot 10^{5} \mathrm{~N} / \mathrm{rad}, 400 \mathrm{kgm}^{2}, \quad 5 \cdot 10^{3} \mathrm{kgm}^{2}$ , $\left.5 \cdot 10^{5} \mathrm{Nm} / \mathrm{rad}, 2 \cdot 10^{4} \mathrm{Nms} / \mathrm{rad}, 0.4 \mathrm{~m}\right]$. The vehicle mass, $m$, and the distances of the front and rear axles from the COG, $a$ and $b$, respectively, were directly measured in the vehicle. Their values are: $m=2150 \mathrm{~kg}, a=1.51 \mathrm{~m}$ and $b=2.04 \mathrm{~m}$.

For the constrained DKF algorithms the lower bounds considered are $L B=\left[10^{3} \mathrm{~N} / \mathrm{rad}, 10^{3} \mathrm{~N} / \mathrm{rad}\right.$, 


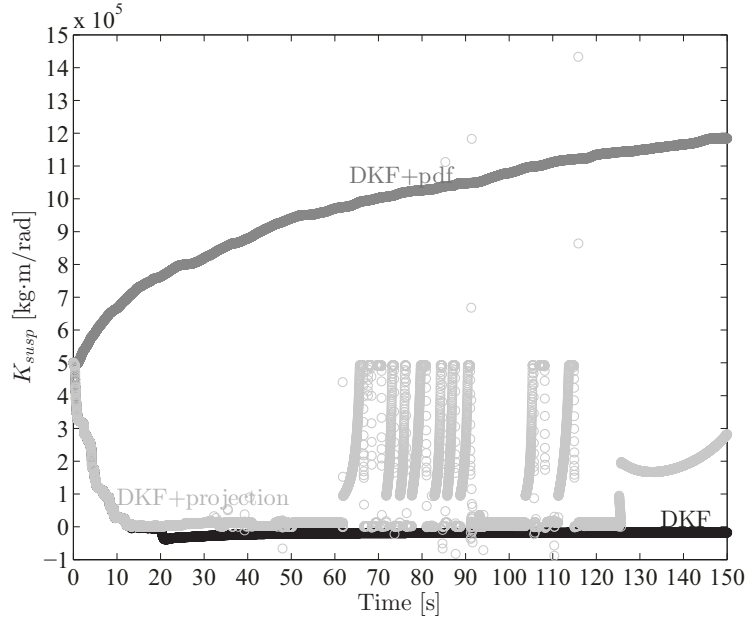

Fig. 11. Roll stiffness estimation: DKF without constraints (black), DKF + modifie projection method (light grey) and DKF + pdf truncation method (dark grey).

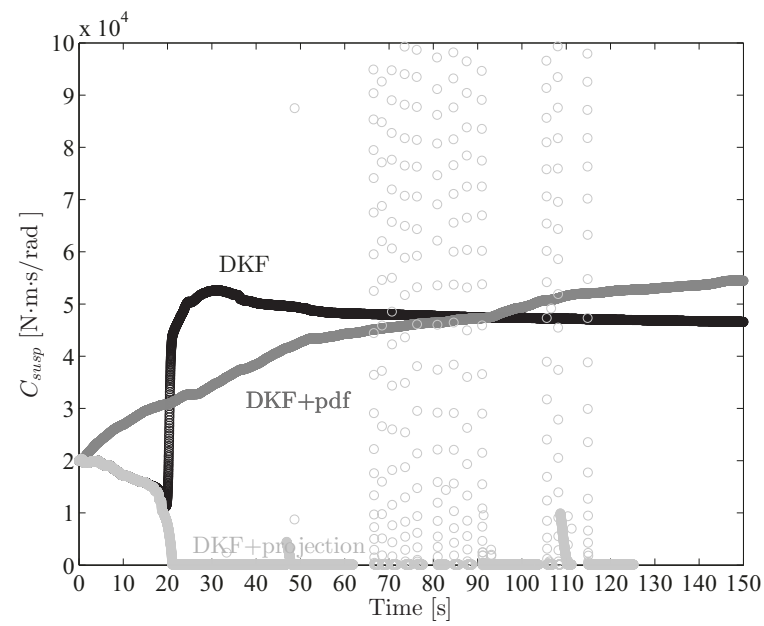

Fig. 12. Roll damping coefficien estimation: DKF without constraints (black), $\mathrm{DKF}+$ modifie projection method (light grey) and DKF + pdf truncation method (dark grey)

$\left.100 \mathrm{kgm}^{2}, 100 \mathrm{kgm}^{2}, 100 \mathrm{Nm} / \mathrm{rad}, \quad 100 \mathrm{Nms} / \mathrm{rad}, \quad 0.1 \mathrm{~m}\right]$ and the upper bounds are $U B=\left[10^{6} \mathrm{~N} / \mathrm{rad}, 10^{6} \mathrm{~N} / \mathrm{rad}, 5 \cdot 10^{3}\right.$ $\left.\mathrm{kgm}^{2}, 10^{4} \mathrm{kgm}^{2}, 5 \cdot 10^{6} \mathrm{Nm} / \mathrm{rad}, 5 \cdot 10^{6} \mathrm{Nms} / \mathrm{rad}, 0.6 \mathrm{~m}\right]$.

The results of estimation of vehicle parameters using the DKF algorithm without constraints are shown in Figures 7-13 (black). The fina values of parameters are:

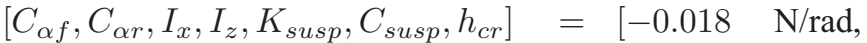
$-0.045 \mathrm{~N} / \mathrm{rad}, 28 \mathrm{kgm}^{2}, 8270 \mathrm{kgm}^{2},-17779.25 \mathrm{Nm} / \mathrm{rad}$, $46570.06 \mathrm{Nms} / \mathrm{rad},-0.83 \mathrm{~m}]$.

In Figures 7, 8, 11 and 13 it can be seen that the approach does not provide a good estimation of parameters because some do not have physical meaning: the values cannot be negative. The estimation of vehicle roll angle $\phi_{v}$ and road bank angle $\phi_{r}$ also is not suitable (see Figure 14). The values obtained for these angles are not realistic, achieving values over 1000 degrees, although the estimation of output state (total roll angle) was very similar to sensor measurements (see Figure 15).

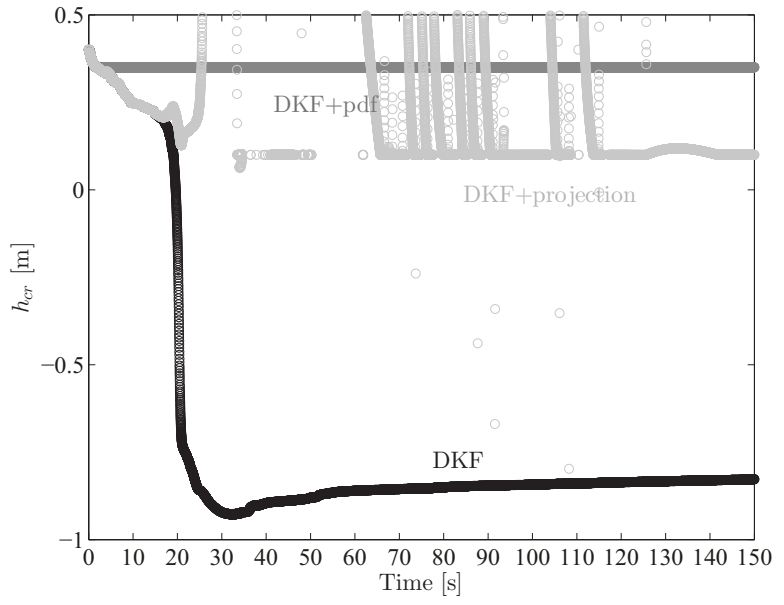

Fig. 13. Distance from roll center to COG estimation: DKF without constraints (black), DKF + modifie projection method (light grey) and DKF + pdf truncation method (dark grey).

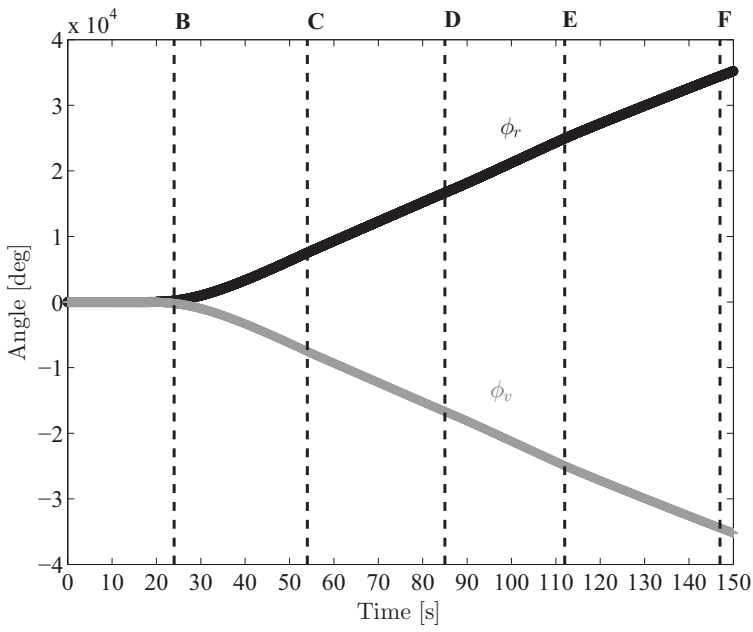

Fig. 14. Vehicle roll angle (grey) and road bank angle estimation (black) using a DKF algorithm without constraints.

The results of vehicle parameters estimation using a constrained DKF based on modifie projection approach are shown in Figures 7-13 (light grey). In this case, the value of the parameters does not converge and the majority of them oscillate between their boundary limits. This method provides an unrealistic road bank angle estimate, with values above 100 degrees, completely out of the physical range.

Finally, the results of vehicle parameters estimation using a constrained DKF based on pdf truncation approach are shown in Figures 7-13 (dark grey). In this case, the fina values of parameters are:

$\left[C_{\alpha f}, C_{\alpha r}, I_{x}, I_{z}, K_{\text {susp }}, C_{\text {susp }}, h_{c r}\right]=[121205 \quad \mathrm{~N} / \mathrm{rad}$, $324885.28 \mathrm{~N} / \mathrm{rad}, \quad 1493.62 \mathrm{kgm}^{2}, \quad 2550.01 \mathrm{kgm}^{2}$, $1184160.47 \mathrm{Nm} / \mathrm{rad}, 54447.87 \mathrm{Nms} / \mathrm{rad}, 0.35 \mathrm{~m}]$.

In comparison with previous methods, the obtained parameters have physical meaning and the values show a stable behaviour.

The estimate of vehicle roll angle $\phi_{v}$ (grey) and road 


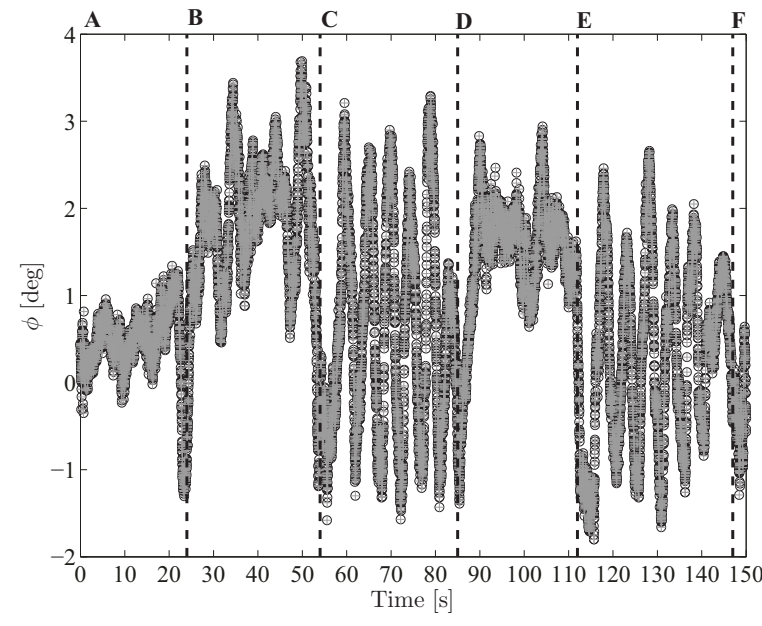

Fig. 15. Sensor measurement of total roll angle (grey) and total roll angle estimation (black) using a DKF algorithm without constraints.

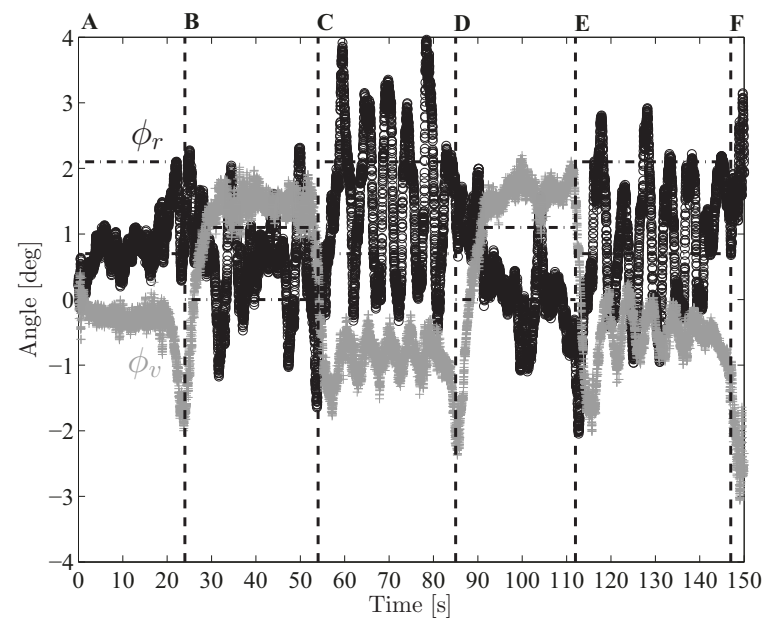

Fig. 16. Vehicle roll angle (grey) and road bank angle (black) estimation using a constrained DKF algorithm based on pdf truncation.

bank angle $\phi_{r}$ (black) is shown in Figure 16. The results of the estimated road bank angle have been validated using a calibrated inclinometer with an accuracy of 0.1 degree. These experimental measurements allow us to set a real range of road bank angles for every manoeuvre. These ranges are, including the uncertainties: [0.7 deg, $2.1 \mathrm{deg}]$ in the straight sections (straight line and slalom manoeuvres) and [0 deg, $1.1 \mathrm{deg}]$ in the constant radius curves (J-turn manoeuvres). These ranges are indicated in Figure 16 with horizontal lines for each stretch of road. In the straight section, the $82 \%$ of the road bank angle estimate data are included between the experimental boundaries limits with a maximum error of 0.5 degrees. However, this percentage is reduced to $60 \%$ considering all sections. It is necessary to take into account the fact that the measurements have been carried out for a heavy vehicle in which the height of COG and the stiffness of tyre greatly affects the vehicle roll angle. These perturbations have an important influenc on the road bank estimation. This effect can be mainly seen during the slalom manoeuvres, where the amplitude of road bank estimation (about 4 degrees) is higher than estimated values for J-turn manoeuvres. In the slalom manoeuvre, the tyre deformation is more affected by rapid dynamic changes.

\section{CONClusion And Future Works}

This paper presents a new method based on a constrained DKF for estimation of vehicle states and parameters. DKF does not work well if the number of states and parameter to estimate is high, so that the consideration of constraints is beneficia in this case. The constraint enforcement method presented in this paper is based on a pdf truncation algorithm that updates the Kalman filte with the mean and covariance of the region of the pdf that lies outside of the constraint hyperplane.

Experimental results show the effectiveness of proposed method and demonstrate that the incorporation of parameters constraints improves its estimation accuracy. In comparison with the DKF method and the DKF with a modifie projection method, the proposed method provides a parameters and states estimation with physical meaning and stable values within the real boundary limits.

Further research could focus on including the non-linear behaviour of the tyre and suspension system. It should improve the vehicle roll angle estimate and, consequently, the road bank estimate.

\section{ACKNOWLEDGMENT}

This work could not have been possible had it not been for the funds provided by the Spanish government through the CICYT project TRA2013-48030-C2-1-R.

\section{REFERENCES}

[1] M. J. L. Boada, B. L. Boada, A. Gauchia, J. A. Calvo, and V. Diaz, "Active roll control using reinforcement learning for a single unit heavy vehicle," Int. J. of Heavy Vehicle Systems, vol. 16, no. 4, pp. 412-430, 2009.

[2] R. Rajamani and D. N. Piyabongkarn, "New paradigms for the integration of yaw stability and rollover prevention functions in vehicle stability control," IEEE Transactions on Intelligent Transportation Systems, vol. 14, no. 1, pp. 249-261, 2013.

[3] N. C. Parida, S. Raha, and A. Ramani, "Rollover-preventive force synthesis at active suspensions in a vehicle performing a severe maneuver with wheels lifted off," Intelligent Transportation Systems, IEEE Transactions on, vol. 15, no. 6, pp. 2583-2594, 2014.

[4] H. Dahmani, O. Pages, A. E. Hajjaji, and N. Daraoui, "Observer-based robust control of vehicle dynamics for rollover mitigation in critical situations," Intelligent Transportation Systems, IEEE Transactions on, vol. 15 , no. 1, pp. 274-284, 2014.

[5] B. Zhu, Q. Piao, J. Zhao, and L. Guo, "Integrated chassis control for vehicle rollover prevention with neural network time-to-rollover warning metrics," Advances in Mechanical Engineering, vol. 8, no. 2, pp. 1-13, 2016.

[6] S.-H. You, J.-O. Hahn, and H. Lee, "New adaptive approaches to realtime estimation of vehicle sideslip angle," Control Engineering Practice, vol. 17, no. 12, pp. 1367-1379, 122009.

[7] J. J. Oh and S. B. Choi, "Vehicle velocity observer design using 6-d imu and multiple-observer approach," IEEE Transactions on Intelligent Transportation Systems, vol. 13, no. 4, pp. 1865-1879, 2012.

[8] A. Katriniok and D. Abel, "Adaptive ekf-based vehicle state estimation with online assessment of local observability," IEEE Transactions on Control Systems Technology, vol. PP, no. 99, pp. 1-14, 2015. 
[9] K. Nam, S. Oh, H. Fujimoto, and Y. Hori, "Estimation of sideslip and roll angles of electric vehicles using lateral tire force sensors through rls and kalman filte approaches," IEEE Transactions on Industrial Electronics, vol. 60, no. 3, pp. 988-1000, 2013.

[10] J. H. Yoon and H. Peng, "Robust vehicle sideslip angle estimation through a disturbance rejection filte that integrates a magnetometer with gps," IEEE Transactions on Intelligent Transportation Systems, vol. 15, no. 1, pp. 191-204, 2014

[11] K. C. Veluvolu, J. J. Rath, M. Defoort, and Y. C. Soh, "Estimation of side slip and road bank angle using high-gain observer and higher-order sliding mode observer," in Recent Advances in Sliding Modes (RASM), 2015 International Workshop on, 2015, pp. 1-6.

[12] H. E. Tseng, "Dynamic estimation of road bank angle," Vehicle System Dynamics, vol. 36, no. 4-5, pp. 307-328, 11/01 2001.

[13] J. Kim, H. Lee, and S. Choi, "A robust road bank angle estimation based on a proportional integral $\mathrm{h}$ infinit filte," Proceedings of the Institution of Mechanical Engineers, Part D: Journal of Automobile Engineering, vol. 226, no. 6, pp. 779-794, June 012012.

[14] J. Lu, D. Messih, and A. Salib, "Roll rate based stability control - the roll stability control system," in The 20th Enhanced Safety Vehicles Conf, 2007.

[15] S. Garatti and S. Bittanti, "Parameter estimation in the pacejka's tyre model through the ts method," in 15th IFAC Symposium on System Identificatio , vol. 15, 6-8 july 2009 2009, pp. 1304-1309.

[16] M. Bolhasani and S. Azadi, "Parameter estimation of vehicle handling model using genetic algorithm," SAE Technical Paper 2002-01-1577, 2002.

[17] E. A. Wan and A. T. Nelson, "Dual kalman filterin methods for nonlinear prediction, smoothing, and estimation," in In Advances in Neural Information Processing Systems 9, 1997.

[18] T. A. Wenzel, K. J. Burnham, M. V. Blundell, and R. A. Williams, "Dual extended kalman filte for vehicle state and parameter estimation," Vehicle System Dynamics, vol. 44, no. 2, pp. 153-171, 02/01 2006.

[19] V. A. Bavdekar, J. Prakash, S. L. Shah, and R. B. Gopaluni, "Constrained dual ensemble kalman filte for state and parameter estimation," in American Control Conference (ACC), 2013, 2013, pp. 3093-3098.

[20] S. R. Burkul, P. R. Pawar, and K. R. Jagtap, "Estimation of vehicle parameters using kalman filter review," Int. J. Curr. Eng. Technol., vol. 4, no. 4, pp. 2731-2735, 2014

[21] S. Hong, C. Lee, F. Borrelli, and J. K. Hedrick, "A novel approach for vehicle inertial parameter identificatio using a dual kalman filte," Intelligent Transportation Systems, IEEE Transactions on, vol. 16, no. 1, pp. 151-161, 2015.

[22] E. Nada, A. Ahmed, and M. Abd-Alla, "Modifie dual unscented kalman filte approach for measuring vehicle states and vehicle parameters," International Journal of Engineering Research Technology, vol. 3, no. 8, pp. 1423-1430, 2014.

[23] D. Simon, Optimal State Estimation: Kalman, $H$ infinit , and Nonlinear Approaches. Wiley, 2006.

[24] C. Yang and E. Blasch, "Kalman filterin with nonlinear state constraints," in Information Fusion, 2006 9th International Conference on, 2006, pp. $1-8$.

[25] D. Simon and T. Chia, "Kalman filterin with state equality constraints," IEEE Transactions on Aerospace and Electronic Systems, vol. 39, pp. 128-136, 2002.

[26] N. Shimada, Y. Shirai, Y. Kuno, and J. Miura, "Hand gesture estimation and model refinemen using monocular camera-ambiguity limitation by inequality constraints," in Automatic Face and Gesture Recognition, 1998. Proceedings. Third IEEE International Conference on, 1998, pp. 268-273.

[27] D.Simon and D.L.Simon, "Aircraft turbofan engine health estimation using constrained kalman filtering" Journal of Engineering for Gas Turbines and Power, vol. 127, pp. 323-328, 2005.

[28] S. Tully, G. Kantor, and H. Choset, "Inequality constrained kalman filterin for the localization and registration of a surgical robot," in Intelligent Robots and Systems (IROS), 2011 IEEE/RSJ International Conference on, 2011, pp. 5147-5152.

[29] J. Ryu and J. C. Gerdes, "Estimation of vehicle roll and road bank angle," in American Control Conference, 2004. Proceedings of the 2004, vol. 3 , 2004, pp. 2110-2115 vol.3.

[30] Y. Sebsadji, S. Glaser, S. Mammar, and M. Netto, "Road bank angle estimation using disturbance observer," in In. Proceedings of AVEC 2002 6th Int. Symposium on Advanced Vehicle Control, 2002, pp. 381-386.

[31] C. Zong, D. Hu, and H. Zheng, "Dual extended kalman filte for combined estimation of vehicle state and road friction," Chinese Journal of Mechanical Engineering, vol. 26, no. 2, pp. 313-324, 03/01 2013.
[32] Racelogic. Vbox 3i dual antenna. measures slip and pitch/roll (rlvb3isl). [Online]. Available: http://www.racelogic.co.uk/_downloads/ vbox/Datasheets/Data_Loggers/RLVB3iSL_Data.pdf

[33] D. Simon and D. L. Simon, "Kalman filterin with inequality constraints for turbofan engine health estimation," IEE Proc.-Control Theory Appl, vol. 153 , no. 3, pp. 371-378, 2006.

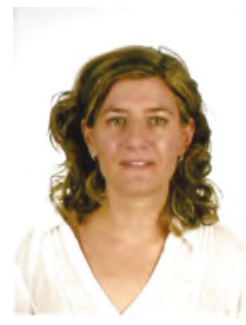

Beatriz L. Boada received her Industrial Engineering and doctoral degrees from Universidad Carlos III de Madrid (UC3M) in 1996 and 2002, respectively. She was a research assistant in the Department of Systems and Automation Engineering at UC3M from 1997 to 2002. She has worked in the localization and mapping problems in the fiel of robotic. Currently, she is a professor in the Mechanical Engineering Department at UC3M. Her research fiel covers intelligent vehicles, both autonomous and motorized, and control of vehicle in vertical and lateral dynamics. She also researches in magnetorheological damper, modelling and estimation of mechanical systems.

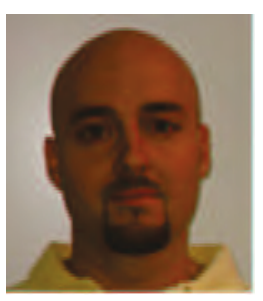

Daniel Garcia-Pozuelo received a B.S. degree in Industrial Engineering with highest honors in 2005 and a doctoral degree in Mechanical Engineering and Industrial Management in 2008 both from UC3M, Spain. He was Honorary Research Fellow in the School of Mechanical Engineering within the College of Engineering and Physical Sciences, University of Birmingham, 2009-2010. He is currently associate professor in the UC3M Mechanical Engineering Department. His research interests include tyre dynamics, intelligent tyre, suspension control and performance, and vehicle stability.

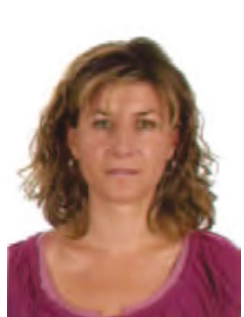

Maria Jesus L. Boada received her Industrial Engineering and doctoral degrees from UC3M in 1996 and 2002, respectively. She was research assistant in the Department of Systems and Automation Engineering at UC3M between 1997 and 2002. She has worked in control architectures and development of learning skills in autonomous mobile robots. She is currently a professor in the UC3M Mechanical Engineering Department. Her research area includes intelligent vehicles, both autonomous and motorized, control of vehicle lateral dynamics and magnetorhe-

ological damper.

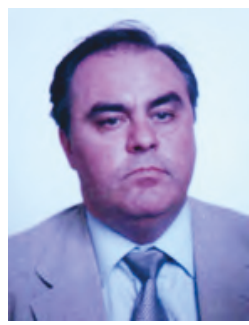

Vicente Diaz is full professor in the Mechanical Engineering Department of UC3M and head of the research group MECATRAN. His area of expertise at UC3M is in the fiel of transports, vehicle dynamics engineering, end-of-life recycling and motor vehicle inspection. He has a large number of publications and papers indexed in JCR in the areas of vehicle dynamic modelling (including heavy vehicle), vehicle lateral control and tyre modelling. He has headed of projects developed for the automobile industry as well as being a leader in tyre research competitive government projects. In addition, he has supervised several $\mathrm{PhD}$ theses on bus structure dynamics, tyre modelling and Electronic Stability Control for automobile vehicles. 